\title{
Building a flagellum in biological outer space
}

\author{
Lewis D. B. Evans, Colin Hughes and Gillian M. Fraser* \\ Department of Pathology, University of Cambridge, Tennis Court Road, Cambridge, CB2 1QP, United Kingdom. \\ * Corresponding Author: G. M. Fraser, Department of Pathology, University of Cambridge, Tennis Court Road; Cambridge, CB2 1QP, \\ United Kingdom; Tel: +44 (0)1223 330245; E-mail: gmf25@cam.ac.uk
}

\begin{abstract}
Flagella, the rotary propellers on the surface of bacteria, present a paradigm for how cells build and operate complex molecular 'nanomachines'. Flagella grow at a constant rate to extend several times the length of the cell, and this is achieved by thousands of secreted structural subunits transiting through a central channel in the lengthening flagellum to incorporate into the nascent structure at the distant extending tip. A great mystery has been how flagella can assemble far outside the cell where there is no conventional energy supply to fuel their growth. Recent work published by Evans et al. [Nature (2013) 504: 287-290], has gone some way towards solving this puzzle, presenting a simple and elegant transit mechanism in which growth is powered by the subunits themselves as they link head-to-tail in a chain that is pulled through the length of the growing structure to the tip. This new mechanism answers an old question and may have resonance in other assembly processes.
\end{abstract}

The structural subunits that comprise the sequential parts of the bacterial flagellum, the 'drive-shaft' rod, flexible hook and semi-rigid helical filament (Figure 1) are unfolded and exported across the cell membrane by a specialised 'Type III' export machinery. Like many active transport processes in the cell, this first phase of export is energised by the hydrolysis of ATP and the proton motive force. On crossing the membrane, the unfolded subunits pass into a narrow $2 \mathrm{~nm}$ diameter central channel, which extends the entire length of the nascent flagellum. Subunits must transit up to $15-20 \mu \mathrm{m}$ in their unfolded state to reach the flagellum tip where they crystallize into the structure aided by a cap 'foldase' (Figure 1c). This subunit transit must of course be energised, as passive diffusion of unfolded subunits would not support the observed constant rate growth of flagella. Yet there is no obvious conventional energy source available in the external channel. So where does the energy come from to power constant rate subunit transit outside the cell?

We set out a series of data that together describe a growth mechanism that is energised intrinsically. Firstly, prior to entering the flagellar central channel, free subunits dock at the cell membrane export machinery, specifically via a conserved subunit targeting motif that binds to an exposed hydrophobic pocket on the machinery 'export gate' component FlhB. Each docked subunit is then sequentially captured from the gate by linking to the free Cterminal 'tail' of the preceding subunit, the $\mathrm{N}$-terminus of which has already entered the flagellar channel (Figure 1a). The $\mathrm{N}$ - and $\mathrm{C}$-terminal helices of these adjacent subunits are predicted to link by forming a parallel coiled-coil (Figure $1 \mathrm{~b}$ ), with each subunit contributing 14-32 residues. Successive linkage allows a subunit chain to form. Each newly-linked subunit is then pulled from the gate into the flagellar channel by the thermal motion of the unfolded subunit chain anchored at its other end at the flagellum tip. Repeated folding of subunits into the growth tip (Figure 1c) not only provides directionality to subunit transit, it also causes the chain to shorten and thus stretch, exerting an increasing pulling force on the next subunit at the gate, eventually pulling it off the gate and into the channel. The vacated export gate is then free to bind a new incoming subunit that again links to the growing chain. In this way, successive rounds of subunit linking at the cell membrane export machinery are coupled to subunit crystallization at the tip to allow continuous subunit transit and constant rate growth of the flagellum.

The proposed chain mechanism imposes a strict prediction on the forces underlying the respective stages of subunit passage, in particular that subunit anchoring at the flagellum tip is stronger than the coiled-coil links between subunits in the chain, which in turn must be stronger than the subunit binding to the export gate. Our thermodynamic analysis confirmed this to be the case, with the estimated force required to break the anchor (Figure 1c) being an

MICROREVIEW on: Evans LDB, Poulter S, Terentjev EM, Hughes C and Fraser GM. (2013) A chain mechanism for flagellum growth. Nature 504: 287-290. doi:10.1038/nature12682 
order of magnitude greater than the force required to break the links between subunits (Figure 1b) in the chain, which in turn is an order of magnitude greater than the force needed to pull subunits from the gate (Figure 1a).

Our proposed chain mechanism gives rise to a number of questions, the first being what happens if the subunit chain breaks e.g. by shearing of the flagellum? We anticipate that breaking the chain would result in a pause in flagellum growth as the most cell-distal subunit in the remaining chain diffuses to the tip where it would fold and create a new anchor to re-establish the chain mechanism. Another aspect is that the subunit chain moving through the channel typically contains a mix of subunits that link head-to-tail e.g. flagellin and filament cap subunits (Figure 1 ), each being required at a different stage of assembly. On reaching the tip, the subunits in the chain that are not needed in the structure will fold as they leave the channel but do not incorporate, being discarded instead. This can be observed as accumulation of, for example, monomeric cap subunits in the extracellular environment. Our analysis suggests that even in the absence of assembly, subunit folding at the tip provides sufficient anchoring for the chain mechanism to function. A further question is whether the chain mechanism might operate in assembly of molecular needles used by pathogens to deliver virulence effectors into eukaryotic host cells? Needles use export machineries that are evolutionarily related to that used by flagella and, as in flagella assembly, unfolded structural subunits are delivered across the bacterial membrane before they transit through a narrow central channel and incorporate at the needle tip. Furthermore, needle subunits have helical termini that could conceivably link as parallel coiledcoils. As seen in flagella growth, export without assembly is a characteristic of effectors secreted through the virulence needles. However, little is known about the dynamics of needle growth, and further investigation is needed to establish whether the chain mechanism operates during the transit of needle structural subunits.

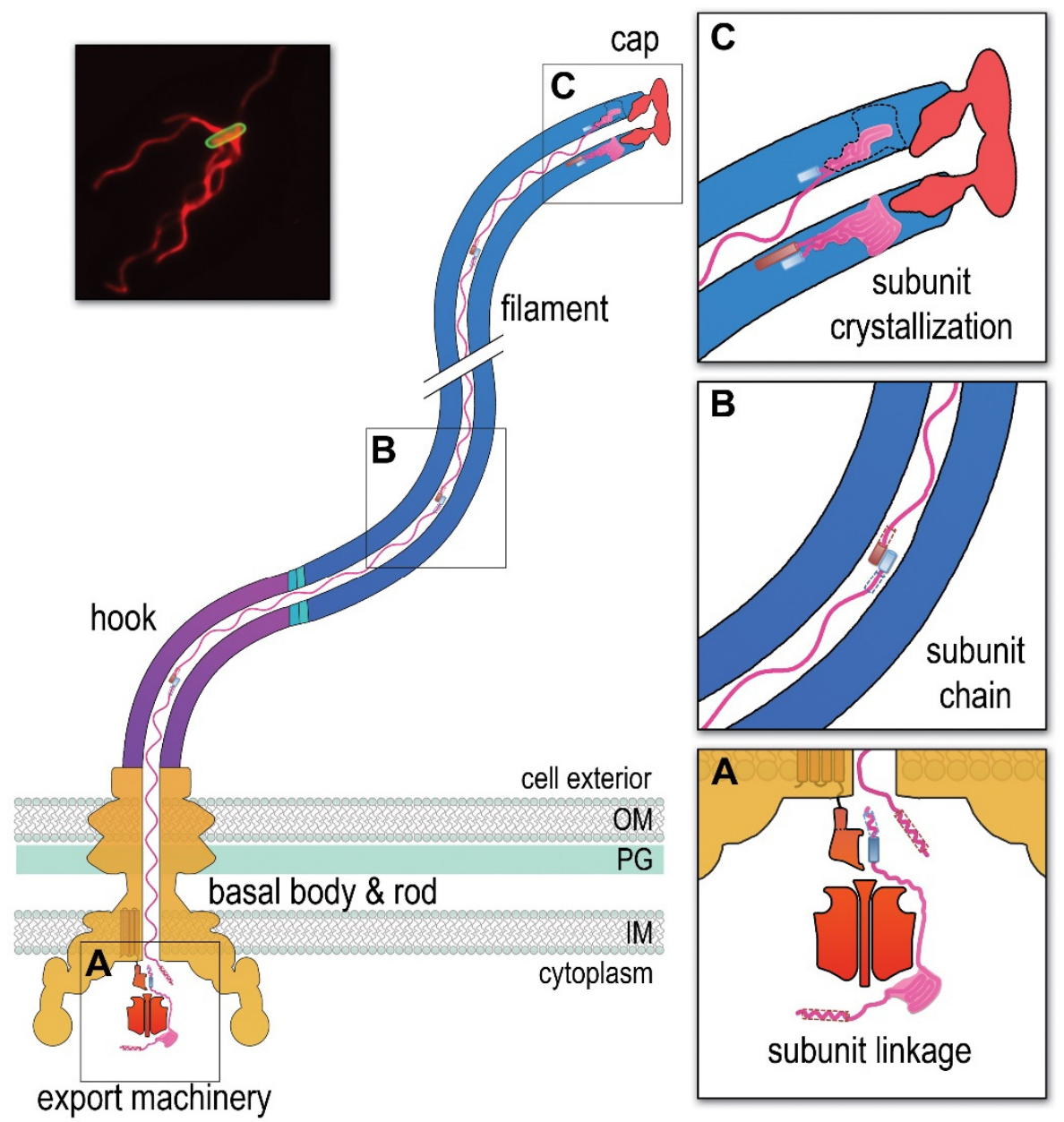

FIGURE 1: A chain mechanism for bacterial flagellum growth. Flagella on the surface of the bacterial cell (inset top right, Salmonella cell membrane stained green and flagella stained red, visualized by epifluorescence microscopy) comprise three contiguous substructures - the basal body, hook and filament - that are assembled sequentially. A central channel runs through these substructures to the flagellum tip. The basal body houses the flagellar export machinery (A) and the rod (FliE, FlgB, FlgC, FlgF and FlgG), which extends from the inner membrane (IM) and crosses the periplasm, peptidoglycan (PG) cell wall and outer membrane (OM). The cell surface hook (FlgE) is a flexible universal joint that connects the external flagellum filament (flagellin, FliC) to the basal body. (A) Subunits of the rod, hook and filament link head-to-tail at the cytoplasmic membrane export machinery. Subunits are first unfolded by the export ATPase complex (red) and then dock at the FlhB export gate (orange). The $\mathrm{N}$ terminal helix of the docked subunit is then captured by the free C-terminal helix of an exiting subunit in the flagellum channel. (B) Sequential subunits are linked head-to-tail in a chain by juxtaposed terminal helices forming parallel coiled-coils. The resulting chain of unfolded subunits is connected through the flagellum central channel to the distal tip of the flagellum. (C) The subunits in the chain transit from the gate to the tip. Subunits fold and incorporate into the flagellum tip beneath cap foldases (FlgJ for rod, FlgD for hook and FliD for filament subunit assembly). Subunit folding and/or crystallization not only provides a strong anchor at the flagellum tip, it also shortens the chain in the channel thus exerting a pulling force on the next subunit at the export gate, pulling it from the gate. The pulling force then drops rapidly as the new unfolded subunit enters the channel. This process repeats for each subunit captured into the chain. 
Our proposed chain mechanism for flagellum growth is supported by experimental evidence, polymer theory and thermodynamic analysis, and we suggest that it explains observations made on how flagella grow. The mechanism describes how a large macromolecular structure can be assembled outside living cells, and may throw new light on other assembly problems and how polymers move through channels, a classic problem in biology and physics.

\section{ACKNOWLEDGMENTS}

This work was supported by a Wellcome Trust Programme Grant (to C.H. and G.M.F.)

\section{CONFLICT OF INTEREST}

The authors declare no competing financial interests.

\section{COPYRIGHT}

(C) 2014 Ewans et al. This is an open-access article released under the terms of the Creative Commons AttributionNonCommercial-Share Alike 3.0 license, which allows readers to twist, transform, or build upon the article and then distribute the resulting work non-commercially, as long as they credit the original authors and license their new creations under the identical terms.

Lewis D. B. Evans present address: Wellcome Trust/Cancer Research UK Gurdon Institute, Henry Wellcome Building, University of Cambridge, Tennis Court Road, Cambridge, CB2 1QN, United Kingdom.

Please cite this article as: Lewis D. B. Evans, Colin Hughes and Gillian M. Fraser (2014). Building a flagellum in biological outer space. Microbial Cell 1(2): 64-66. doi: 10.15698/mic2014.01.128 